\title{
Immunohistochemical expression of $\pi$ class glutathione $S$-transferase in the basal cell layer of benign prostate tissue following chronic treatment with finasteride
}

Rodolfo Montironi, Roberta Mazzucchelli, Roberto Pomante, Deborah Thompson, Vinicius Duval da Silva, Linda Vaught, Peter H Bartels

\begin{abstract}
Background-Glutathione S-transferases (GST) may prevent carcinogenesis through inactivation of reactive electrophiles by conjugation to reduced glutathione. Treatment directed at the induction or preservation of GST- $\pi$ expression in normal epithelium could have a profound impact on the prevention of prostate neoplasia. Finasteride, a 5- $\alpha$ reductase inhibitor, is used as a chemopreventive agent because it blocks the conversion of testosterone to its byproduct which promotes prostate tumour growth. Objective-To investigate GST- $\pi$ expression immunohistochemically in benign prostate tissue from untreated patients and from patients chronically treated with finasteride.
\end{abstract}

Materials-Immunostaining with antiGST- $\pi$ antibody was performed on 10 (cysto-) prostatectomy, eight simple prostatectomy, and three transurethral prostatectomy specimens. The first set of 10 prostates was from untreated patients operated on for bladder cancer. The other cases were from patients with benign prostatic hyperplasia and chronically treated with finasteride. None of the specimens in either group showed prostatic cancer, prostatic intraepithelial neoplasia, urothelial carcinoma, or chronic prostatitis. Specimens were evaluated for the presence, intensity, and distribution of immunostaining.

Results-Diffuse cytoplasmic immunostaining was observed in the basal cell layer of the untreated specimens. Some variability in the expression of GST- $\pi$ was seen within each zone and also between the prostate zones. Only a minority of the secretory cells was stained weakly, mainly in the subnuclear region of the cells facing an uninterrupted basal cell layer. Staining was more homogeneously diffuse in the cytoplasm of the luminal cells facing the basement membrane directly. In the benign epithelium of the finasteride treated specimens the circumferential staining of the basal cells appeared to be more continuous than in the untreated cases, the gaps in the stained basal cell layer being fewer, shorter, or even absent in some ducts and acini. There was no variability in the intensity of staining of the basal cell layer, all the cells being intensely stained in a uniform way. The intensity of staining of the secretory cells was not influenced by finasteride treatment.

Conclusions-Following chronic treatment with finasteride the immunohistochemical expression of $\pi$ class glutathione S-transferase in the benign prostate ducts and acini is upregulated in relation to an expanded basal cell layer. This could indicate that finasteride acts as a GST- $\pi$ inducer.

(F Clin Pathol 1999;52:350-354)

Keywords: prostate; benign prostatic hyperplasia; finasteride; $\pi$ class glutathione $S$-transferase

Glutathione S-transferases (GST) are a family of multifunctional isoenzymes that catalyse the nucleophilic addition of glutathione to a widely heterogeneous groups of compounds. ${ }^{1}$ GST isoenzymes can be characterised on the basis of substrate specificity, sensitivity to inhibitors, isoelectric points, immunological cross reactivity, and amino acid composition and sequence..$^{2-5}$ Various biological functions have been ascribed to this enzyme group, including intracellular binding and transport of lipophilic compounds such as bile products, steroid hormones, drugs, and xenobiotics. ${ }^{6}$ By far the most studied function of the GST enzymes is their role in cellular detoxification, ${ }^{7}$ primarily against oxygen free radicals and peroxides produced by physiological cellular processes and exogenous stimuli. GST may prevent carcinogenesis through inactivation of reactive electrophiles by conjugation to reduced glutathione. ${ }^{8}$ Human GST have been classified into five families: $\alpha, \mu, \theta, \pi$, and microsomal. ${ }^{19}$ The most widely investigated with respect to human malignancy is GST- $\pi .^{8}{ }^{10-18}$

Recently it was reported that the regulatory sequences near the gene GSTP1, which encodes the human $\pi$ class GST, commonly appear to be hypermethylated during prostatic carcinogenesis..$^{19}$ In addition, it has been noted that immunohistochemical staining with antiGST $-\pi$ antibodies failed to detect the enzyme in most prostatic adenocarcinomas, despite the presence of abundant staining in normal prostatic epithelial cells and in cells making up benign proliferative prostatic lesions. The implications of this study include identification of GST- $\pi$ as the most common genetic 
alteration in the formation of prostate cancer. ${ }^{19}$ It was shown that total androgen ablation does not restore the expression of this enzyme, indicating that the genetic defect detected in prostate cancer is not reversible under androgen manipulation. ${ }^{20}$

Cookson et $a l^{20}$ and Lee et $a l^{19}$ suggested that treatments directed at the preservation or induction of the activity of this enzyme in normal prostate could have a profound impact on the possible prevention of prostate cancer and its precursors. Finasteride (Proscar ${ }^{\circledR}$; a $5-\alpha$ reductase inhibitor) blocks the conversion of testosterone to dihydrotestosterone, a byproduct which promotes prostate tumour growth. ${ }^{21}$ The availability of drugs like finasteride, capable of suppressing dihydrotestosterone levels without significantly altering testosterone dependent function, has prompted the US National Cancer Institute to propose a large chemopreventive study using this agent. ${ }^{22}{ }^{23}$ No information is currently available on the effect of this drug on the expression of GST- $\pi$.

Our aim in this study was to investigate GST- $\pi$ expression immunohistochemically in benign prostate tissue from untreated patients and from patients chronically treated with finasteride.

\section{Methods}

SPECIMENS

Clinical and morphological data on 10 untreated and 11 finasteride treated prostate specimens were obtained from the files of the Institute of Pathological Anatomy and Histopathology of the University of Ancona. The ages of the patients in both groups ranged between 60 and 70 years. Their serum prostate specific antigen concentrations were less than $4.00 \mathrm{ng} / \mathrm{ml}$.

The untreated control group was composed of patients who had urothelial cell carcinoma of the urinary bladder and had undergone cystoprostatectomy without receiving chemotherapy, hormone treatment, or radiation therapy before surgery. Benign prostatic hyperplasia was present in three patients. The prostatectomy specimens (prostate and seminal vesicles) were separated from the bladder at the level of the bladder neck, covered with India ink, and fixed for 48 hours in neutral buffered formalin (4\%). After fixation, the distal (apical), proximal (basal), and seminal vesicles were removed and submitted for histological examination. The prostate was then step sectioned at $0.4 \mathrm{~cm}$ intervals perpendicular to the long axis (apical to basal) of the gland.

The second group of patients all had benign prostatic hyperplasia and were treated before surgery with $5 \mathrm{mg}$ of finasteride daily for six months. In none of these patients was a biopsy available that had been taken before the start of the treatment. Eight underwent simple prostatectomy and three had transurethral resection of the prostate, owing to the lack of clinical relief of their urethral obstruction. The specimens were fixed for 24 hours in neutral buffered formalin $(4 \%)$. The prostatic adenomas were then cut into $0.4 \mathrm{~cm}$ thick slices.
The cut specimens were postfixed for an additional 24 hours and then dehydrated in graded alcohols, cleared in xylene, embedded in paraffin, and examined histologically as $5 \mu \mathrm{m}$ thick sections. The cross sections of both groups were analysed histologically as whole mount sections. Using haematoxylin and eosin stained sections, one of our team (RM) reviewed all the histological slides, checked the quality of the material, and selected the slides for immunohistochemistry. None of the patients in either group had histological evidence of prostatic cancer, prostatic intraepithelial neoplasia, urothelial carcinoma originating from the urethra, or chronic prostatitis. In this study the central and peripheral zone are also referred to together as the outer prostate or "non-transition zone." A limited amount of peripheral and central zone tissue was identifiable histologically in all the simple prostatectomy specimens in the finasteride treated group.

\section{IMMUNOHISTOCHEMISTRY}

Immunohistochemistry was performed on 5 $\mu \mathrm{m}$ thick paraffin embedded tissue sections on poly-L-lysine coated glass slides. The sections were dried, deparaffinised, and rehydrated. The sections were then pretreated with $0.3 \%$ hydrogen peroxide in methanol for 30 minutes. After an initial 20 minute incubation with $2 \%$ normal goat serum, the specimens were incubated overnight at $4^{\circ} \mathrm{C}$ with primary rabbit polyclonal anti-GST- $\pi$ antibody at a dilution of 1/500 (Delta Biologicals). Following washings in stock phosphate buffered saline (PBS), secondary antibody (Biotinylated antirabbit IgG, at a dilution of 1/500; Vector Laboratories) was applied to the sections and incubated for 60 minutes, followed by washing and incubation with an avidin-biotin-horseradish peroxidase complex (Vector Laboratories; reagents prepared according to the manufacturer's specifications) for 45 minutes. The sections were then washed in two cycles of stock PBS (five minutes each), and the peroxidase reaction developed using 3,3'-diaminobenzidine tetrahydrochlorate (DAB) diluted at $1 \%$ in PBS. Sections were incubated with this solution for five minutes and then thoroughly washed in water. The sections were differentiated with acid alcohol, washed in tap water and then in blue ammonia water, counterstained with a light haematoxylin and eosin, and dehydrated with three changes of $95 \%$ absolute ethanol and xylene (two minutes each). The sections were then cover-slipped and mounted. Tissue sections from normal human liver containing hepatic bile ducts were used as positive controls, while omission of primary antibodies on prostatic and liver sections served as a negative control.

All the sections were examined by light microscopy to assess qualitatively the presence or absence of immunostaining, its intensity of positivity or strength, and its distribution. Among the tissues which stained positive, a distinction was made between staining of the basal cell layer and the secretory acinar epithelium. 

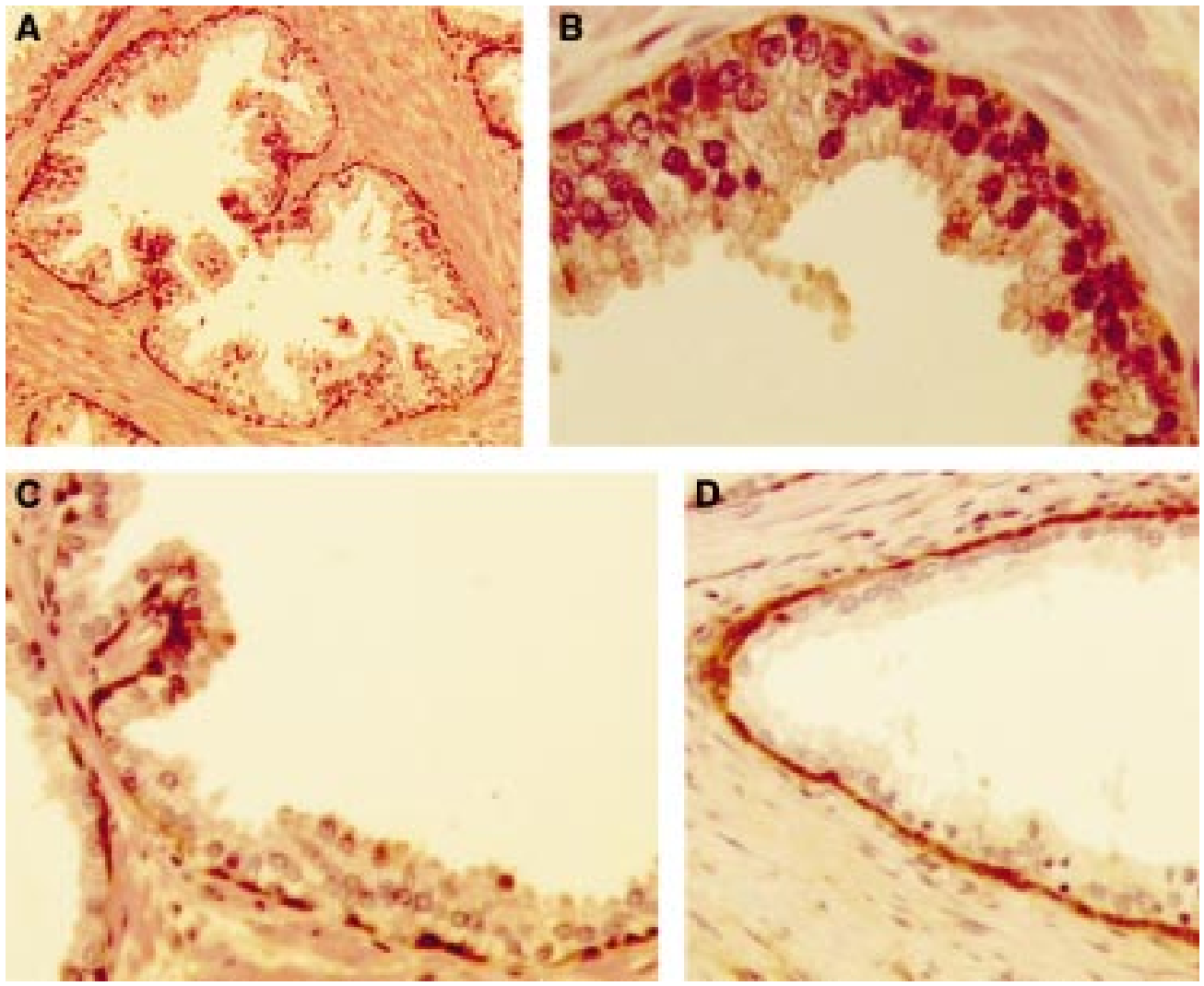

Figure 1 (A) Peripheral zone of the untreated prostate. The GST- $\pi$ antibody primarily stains the cytoplasm. Immunostaining is strong in the basal cell layer and weak in the secretory cell laver (haematoxylin and eosin counterstain, magnification $\times 170$ ). (B) Central zone of the untreated prostate. The immunohistochemical staining is stronger and more diffuse in the basal cell layer than in the luminal (or secretory) cell layer (haematoxylin and eosin counterstain, magnification $\times 170$ ). (C) Transition zone of the untreated prostate. Short gaps in the immunostained basal cell layer were present (haematoxylin and eosin counterstain, magnification $\times 170$ ). (D) Prostate tissue from a patient chronically treated with finasteride. The basal cell layer is easily identifiable as a continuous ring of strongly GST- $\pi$ stained cells (haematoxylin and eosin counterstain, magnification $\times 170$ ).

\section{Results}

The GST- $\pi$ antibody primarily stained the cytoplasm of the cells lining the ducts and acini. In general, staining was stronger and more diffuse in the basal cell layer than in the luminal (or secretory) cell layer (fig $1 \mathrm{~A}$ and 1B). Short gaps in the immunostained basal cell layer were present (fig 1C). Some variability in the expression of GST- $\pi$ in the benign ducts and acini was seen within each zone and also between the prostate zones. In no instance was secretory layer staining present in the absence of basal layer staining. In the luminal cells, positivity was mainly seen in the subnuclear region of those cells facing an uninterrupted basal cell layer, and was more homogeneously diffuse in the cytoplasm of those cells directly facing the basement membrane and the stroma. There was no appreciable difference of intensity in the secretory cells either facing or not facing an intact basal cell layer. No plasma membrane staining was observed. Focal and weak nuclear staining was present in the basal epithelial layer. The prostatic stroma was not immunoreactive, whereas the urothelium and squamous metaplastic epithelium were. Rare cells undergoing mitotic division were seen and did not express GST- $\pi$. There were no differences between the samples with benign prostatic hyperplasia and those of normal tissue from patients unaffected by this disease.

In the finasteride group, two types of ducts and acini were observed. These represent successive steps of involution. Their proportion differed from case to case and, within each case, from field to field. The first type was represented by ducts and acini of approximately the same size as those in the untreated group, though without undulations of the epithelial border. The pattern of staining was similar, the main difference being that the gaps in the stained basal cell layer were either fewer or shorter. The other type was represented by ducts and acini smaller than the former and usually round in shape. The basal cell layer was easily identifiable at low magnification because it was composed of a continuous ring of strongly stained cells (fig 1D). In addition the cells showed nuclei that did not appear elongated and slender as in the first type, but rounder and occasionally plump and crowded. There was no variability in the intensity of staining of the basal cell layer, all the cells being intensely stained in a uniform way. The strength of staining in the secretory cells was not influenced by the treatment. Rare apoptotic cells were seen in both types of ducts and acini, and did not express GST- $\pi$. In both groups of patients, the luminal cell staining was 


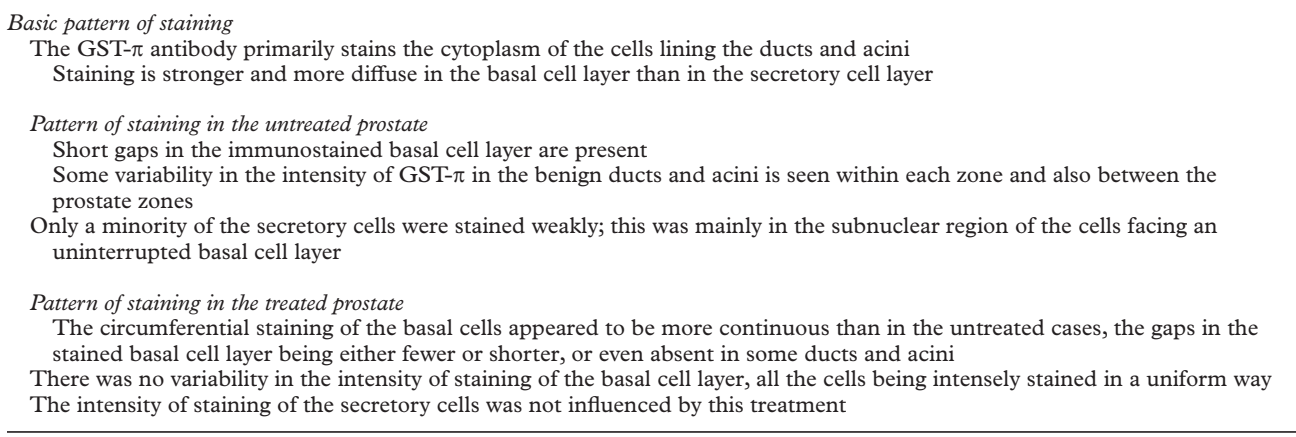

increased in the presence of chronic inflammation (table 1).

\section{Discussion}

The expression of GST- $\pi$ in normal prostate tissue specimens, including those from patients with benign prostatic hyperplasia, has been investigated in previous studies. ${ }^{235} 19202425 \mathrm{In}$ those reports, normal prostatic tissue showed intense cytoplasmic staining in the basal epithelial cells. These findings were confirmed in our present study. Cookson et $a l^{20}$ and Lee et $a l^{19}$ also noted that some normal luminal cells stained weakly for GST- $\pi$, while most contained no detectable enzyme. In the benign tissues from our study, while some degree of staining was seen in the secretory epithelium in almost all cases, the proportion of positive cells was very small. In addition, the pattern of positivity was such that the staining was mainly in the subnuclear region of the cells facing an intact basal cell layer and homogeneously diffuse in those secretory cells facing the basement membrane directly. Although most of the GST- $\pi$ was cytosolic, nuclear staining was reported by Moskaluk et $a l,{ }^{25}$ who found that approximately $50 \%$ of cases also showed focal nuclear staining, primarily in the basal epithelial layer. Our findings on the nuclear staining pattern were similar to those reported by Moskaluk et al. ${ }^{25}$

The purpose of previous immunohistochemical work, either on normal prostate tissue or on benign prostatic hyperplasia, was to establish the reference expression of this enzyme for its subsequent evaluation in prostatic intraepithelial neoplasia and cancer. ${ }^{2620}$ The zone of origin of the normal prostate tissue was not always specified. No attempt was made to compare the findings of the transition zone, either affected or not affected by benign prostatic hyperplasia, with the expression of GST- $\pi$ in the other prostate zones. In our study there was some variability in the strength and proportion of staining of benign acini within each of the three zones and also between the prostate zones. However, the staining of the basal cell layer in the transition zone was qualitatively similar to that of the non-transition zone. No substantial differences were seen between the samples with benign prostatic hyperplasia and those derived from patients unaffected by this disease. Our findings on benign prostatic hyperplasia are in agreement with the data derived from a biochemical study performed by Tew et al. ${ }^{17}$

Finasteride causes intraprostatic dihydrotestosterone levels to decrease by more than $90 \%$, whereas intraprostatic testosterone levels increase by a small factor. ${ }^{21}$ This drug was approved by the United States Food and Drug Administration in 1992 for the treatment of benign prostatic hyperplasia. The Division of Cancer Prevention and Control of the National Cancer Institute started a Prostate Cancer Prevention Trial in 1993. ${ }^{23}$ The hypothesis of the trial is that a prolonged finasteride induced decrease in androgenic stimulation of the prostate will inhibit or retard the progression of prostate cells through the carcinogenic process, resulting in a decreased prevalence of diagnosed prostate cancer.

Some of the prostate samples in the present study were from men treated with finasteride before simple prostatectomy or transurethral resection of the prostate was performed. Morphologically, the involution effects induced by finasteride on the epithelial cells were of a minor degree in comparison with the findings usually present following total androgen ablation. ${ }^{26}{ }^{27}$ This is linked to the fact that testosterone, being less active than dihydrotestosterone, still exerts some effect on the epithelium. Finasteride blocks the proliferative activity of the epithelium rather than inducing severe cell loss through apoptosis.

In the current study it was seen that the immunostaining of GST- $\pi$ appeared to increase following treatment. This finding gives support to the view that hormonal manipulation, when finasteride is involved, affects not only the preservation of enzyme expression but also its induction. This is at odds with the results of an investigation made by Cookson et al on the expression of GST- $\pi$ in benign prostatic tissue associated with hormonally treated prostatic carcinoma. ${ }^{20}$ Since they failed to show differences in the proportion of positive cells between treated and untreated normal looking tissue, they concluded that total androgen ablation treatment has no effect on the GST- $\pi$ activity in the normal prostate. They questioned its role in the malignant transformation of prostatic acinar cells. The discrepancy between the data presented by Cookson $e t a l^{20}$ and our results is caused by the fact that Cookson and his colleagues investigated the proportion of positive cells without 
considering the degree of circumferential staining of the basal cell layer. Our approach takes account of the changes in the gaps observed among the basal cells which always express GST- $\pi$.

In conclusion, the immunohistochemical expression of $\pi$ class glutathione S-transferase appears to be upregulated in the basal cell layer of benign prostate ducts and acini following chronic treatment with finasteride. We speculate that the chemopreventive effect of this drug could also be mediated by the induced activity of GST- $\pi$ in the basal cells, which exert a protective influence on the secretory cells. When the basal cells are not present, this role might be taken on by the secretory cells. Quantitative analytical studies employing image analysis systems are in progress and should provide us with additional information on the activity of GST- $\pi$.

This research has been supported by a grant from the University of Ancona and, in part, by a grant from the National Cance Institute, R 35 CA 53877 (PHB). The content of this paper is solely the responsibility of the authors and does not necessarily represent the official views of the University of Ancona and the National Cancer Institute.

1 Campbell JAH, Corrigall AV, Guy A, et al. Immunohistologic localization of alpha, mu, and pi class glutathione S-transferases in human tissues. Cancer 1991;67:1608-13.

2 Canada AT, Roberson KM, Vessella RL, et al. Glutathione and glutathione S-transferase in benign and malignant prostate cell lines and prostate tissue. Biochem Pharmacol 1996;51:87-90.

3 Di Ilio C, Aceto A, Bucciarelli $\mathrm{T}$, et al. Glutathione transferase isoenzymes from human prostate. Biochem $\mathcal{F}$ 1990;271:481-5.

4 Moscow JA, Fairchild CR, Madden MJ, et al. Expression of anionic glutathione-S-transferase and p-glycoprotein genes in human tissue and tumors. Cancer Res 1989;49:1422-8.

5 Terrier P, Townsend AJ, Coindre JM, et al. An immunohistochemical study of pi class glutathione S-transferase expression in normal human tissue. Am F Pathol 1990;137 845-53.

6 Murray GI, Taylor VE, McKey JA, et al. The immunohistochemical localization of drug-metabolizing enzymes in prostate cancer. F Pathol 1995;177:147-52.

7 Ripple M, Mulcahy RT, Wilding G. Characteristics of the glutathione/glutathione-S-transferase detoxification system in melphalan resistant human prostate cancer cells. $尹$ Uro 1993;150:209-14.

8 Toffoli G, Viel A, Tumiotto L, et al. Expression of glutathione-S-transferase- $\pi$ in human tumours. Eur $\mathcal{F}$ Cancer 1992;28:1441-6.
9 Mannervik B, Awasthi YC, Board PG, et al. Nomenclature for human glutathione transferases. Biochem $\mathcal{F}$ 1992;282: 305-8.

10 Cairns J, Wright C, Cattan AR, et al. Immunohistochemical demonstration of glutathione S-transferases in primary human breast carcinomas. F Pathol 1992;166:19-25.

11 Green JA, Robertson LJ, Clark AH. Glutathione $S$-transferase expression in benign and malignant ovarian tumours. Br F Cancer 1993;68:235-9.

12 Hirata S, Odajima T, Kohama GI, et al. Significance of glutathione S-transferase- $\pi$ as a tumor marker in patients with oral cancer. Cancer 1992;70:2381-7.

13 Niitsu Y, Takahashi Y, Saito T, et al. Serum glutathione-Stransferase- $\pi$ as a tumor marker for gastrointestinal malignancies. Cancer 1989;63:317-23.

14 Oude Ophuis MB, Van Lieshout EMM, Roelofs HMJ, et al. Glutathione S-transferase M1 and T1 and cytochrome P4501A1 polymorphisms in relation to the risk for benign and malignant head and neck lesions. Cancer 1998;82:93643.

15 Randall BJ, Angus B, Akiba R, et al. Glutathione S-transferase (placental) as a marker of transformation in the human cervix uteri: an immunohistochemical study. $B r$ 7 Cancer 1990;62:614-18.

16 Shiratori Y, Soma Y, Maruyama H, et al. Immunohistochemical detection of the placental form of glutathione S-transferase in dysplastic and neoplastic human uterine cervix lesions. Cancer Res 1987;47:6806-9.

17 Tew KD, Clapper ML, Greenberg RE, et al. Glutathione S-transferase in human prostate. Biochim Biophys Acta 1987;926:8-15

18 Thomas DJ, Birch PJ, Vickers J, et al. Glutathione-Sransferase expression in transitional cell carcinoma of the bladder. Br f Urol 1993;72:740-3.

19 Lee WH, Morton RA, Epstein JI, et al. Cytidine methylation of regulatory sequences near the $\pi$-class glutathione S-transferase gene accompanies human prostatic carcinogenesis. Proc Natl Acad Sci USA 1994;91:11733-7.

20 Cookson MS, Reuter VE, Linkov I, et al. Glutathione S-transferase pi (GST- $\pi$ ) class expression by immunohistochemistry in benign and malignant prostate tissue. 7 Urol 1997;157:673-6.

21 Stoner E. 5 $\alpha$-Reductase inhibitors/finasteride. Prostate 1996; 6(suppl):82-7.

22 Bostwick DG, Dundore PA. Biopsy of the prostate. London: Chapman and Hall, 1997.

23 Brawley OW, Thompson IM. Chemoprevention of prostate cancer and the prostate cancer prevention trial. In: Schroeder FH. Recent advances in prostate cancer and benign prostatic hyperplasia. New York: Parthenon, 1997: 51-9.

24 Baker AM, Oberley LW, Cohen MB. Expression of antioxidant enzymes in human prostatic adenocarcinoma. Prostate 997;32:229-33.

25 Moskaluk CA, Duray PH, Cowan KH, et al. Immunohistochemical expression of $\pi$-class glutathione $S$-transferase is down-regulated in adenocarcinoma of the prostate. Cancer 997;79:1595-9.

26 Montironi R, Diamanti L. Morphologic changes in benign prostatic hyperplasia following chronic treatment with a 5 - $\alpha$-reductase inhibitor finasteride. Comparison with combination endocrine therapy. F Urol Pathol 1996;4:123-35.

27 Montironi R, Valli M, Fabris G. Treatment of benign prostatic hyperplasia with a 5 - $\alpha$-reductase inhibitor (finasteride). Morphological evaluation in patients who failed to respond. F Clin Pathol 1996;49:324-8. 